\title{
Analytic treatment of near-extremal charged black holes supporting non-minimally coupled massless scalar clouds
}

\author{
Shahar Hod ${ }^{1,2, a}$ \\ ${ }^{1}$ The Ruppin Academic Center, Emeq Hefer 40250, Israel \\ 2 The Hadassah Academic College, 91010 Jerusalem, Israel
}

Received: 12 November 2020 / Accepted: 29 November 2020 / Published online: 14 December 2020

(C) The Author(s) 2020

\begin{abstract}
It has recently been revealed that massless scalar fields which are non-minimally coupled to the Maxwell electromagnetic tensor can be supported in the exterior spacetime regions of spherically symmetric charged black holes. The boundary between scalarized charged black-hole spacetimes and bald (scalarless) Reissner-Nordström black holes is determined by the presence of a critical existence-line which describes spatially regular linearized scalar 'clouds' that are supported in the black-hole spacetime. In the present paper we use analytical techniques in order to solve the Klein-Gordon wave equation for the non-minimally coupled linearized scalar fields in the spacetimes of near-extremal supporting black holes. In particular, we derive a remarkably compact analytical formula for the discrete resonant spectrum $\{\alpha(l, Q / M ; n)\}_{n=1}^{n=\infty}$ which characterizes the dimensionless coupling parameter of the composed ReissnerNordström-black-hole-nonminimally-coupled-masslessscalar-field configurations along the critical existence-line of the Einstein-Maxwell-scalar theory (here $Q / M$ is the dimensionless charge-to-mass ratio of the central supporting black hole and $l$ is the angular harmonic index of the supported scalar configurations).
\end{abstract}

\section{Introduction}

The mathematically elegant no hair theorems presented in [18] have revealed the physically important fact that spatially regular static massless scalar field configurations (scalar 'hairy' configurations) cannot be supported in spherically symmetric charged black-hole spacetimes.

Intriguingly, it has recently been proved in the physically important studies $[9,10]$ that scalar fields which are nonminimally coupled to the Maxwell electromagnetic tensor can be supported in asymptotically flat charged black-hole

a e-mail: shaharhod@gmail.com (corresponding author) spacetimes. In particular, it has been revealed in $[9,10]$ that, in composed Einstein-Maxwell-scalar theories whose actions contain a direct interaction term between a function $f(\phi)$ of the scalar field and the electromagnetic Maxwell invariant $F_{\mu \nu} F^{\mu \nu}$, charged black holes with spatially regular horizons can support external configurations of the non-minimally coupled massless scalar fields. This physically intriguing phenomenon is known by the name black-hole spontaneous scalarization.

A physically motivated requirement in any EinsteinMaxwell-scalar theory is that the bald Reissner-Nordström black-hole spacetime should be a valid solution of the field equations in the limiting trivial $\phi \equiv 0$ case. It has been proved in $[9,10]$ that this requirement implies that the scalar coupling function $f(\phi)$ should be characterized by the universal weakfield behavior $f(\phi)=1+\alpha \phi^{2}+O\left(\phi^{4}\right)$, where the physical parameter $\alpha$ is the dimensionless scalar-Maxwell coupling constant of the theory.

The boundary between hairy (scalarized) charged blackhole spacetimes and the bald Reissner-Nordström black-hole spacetime in the non-minimally coupled Einstein-Maxwellscalar theory is determined by a critical existence-line $\alpha_{\text {critical }}=\alpha(Q / M ; l)[9-12]$. This boundary line describes charged Reissner-Nordström black holes that support spatially regular linearized configurations of the non-minimally coupled scalar fields (the term scalar 'clouds' is usually used in the physics literature [13-16] in order to describe the linearized hairy scalar configurations that sit on the critical existence-line of a field theory).

Interestingly, it has been nicely demonstrated in $[9,10]$ that, for a given value $l$ of the scalar angular harmonic index, the critical existence-line $\alpha_{\text {critical }}=\alpha(Q / M ; l)$ of the physical system, which separates bald Reissner-Nordström black holes from scalarized black-hole solutions of the coupled Einstein-Maxwell-scalar field equations, is universal in the sense that different non-linear scalar coupling functions $\{f(\phi)\}$ that satisfy the physically motivated relation 
$f(\phi)=1+\alpha \phi^{2}+O\left(\phi^{4}\right)[9,10]$ lead to the same critical existence-line in the linearized weak-field regime of the composed physical system.

The important physical results presented in $[9,10]$ have revealed that, for spherically symmetric scalar clouds (which are characterized by $l=0$ ), the critical existence-line $\alpha_{\text {critical }}=\alpha(Q / M ; l=0)$ of the composed EinsteinMaxwell-scalar theory is determined by the zeros of the hypergeometric function [see Eq. (44) below]. The corresponding functional behavior $\alpha_{\text {critical }}=\alpha(Q / M ; l=0)$ of the $l=0$ critical existence-line of the charged-black-holenonminimally-coupled-massless-scalar-field system has been determined analytically in [17] for near-extremal (1 $Q / M \ll 1)$ central supporting black holes. To the best of our knowledge, the critical existence-lines of $n o n$-spherically symmetric scalar clouds have thus far not been determined analytically in the physics literature.

The main goal of the present paper is to explore the physical and mathematical properties of non-spherically symmetric scalar clouds. In particular, we shall explicitly prove below that, for near-extremal charged supporting black holes, the functional behavior $\alpha_{\text {critical }}=\alpha(Q / M ; l)$ of the critical existence-lines that characterize the composed charged-black-hole-nonminimally-coupled-masslessscalar-field system can be determined analytically.

\section{Description of the system}

We shall analyze the physical and mathematical properties of composed near-extremal-Reissner-Nordström-blackhole-nonminimally-coupled-massless-scalar-field configurations along the critical existence-line which characterizes the Einstein-Maxwell-scalar theory. The spacetime of the charged supporting black hole is described by the curved line element [18]

$d s^{2}=-h(r) d t^{2}+\frac{1}{h(r)} d r^{2}+r^{2}\left(d \theta^{2}+\sin ^{2} \theta d \phi^{2}\right)$,

where

$h(r)=1-\frac{2 M}{r}+\frac{Q^{2}}{r^{2}}$.

Here the physical parameters $\{M, Q\}$ are respectively the asymptotically measured mass and electric charge of the black-hole spacetime. The radii

$r_{ \pm}=M \pm \sqrt{M^{2}-Q^{2}}$

of the (outer and inner) black-hole horizons are the characteristic zeros of the metric function $h(r)$.
The non-minimally coupled Einstein-Maxwell-scalar theory is characterized by the action $[9,10]$

$S=\int d^{4} x \sqrt{-g}\left[R-2 \nabla_{\alpha} \phi \nabla^{\alpha} \phi-f(\phi) \mathcal{I}\right]$,

where the electromagnetic source term $\mathcal{I}$ of the charged spacetime is given by

$\mathcal{I}=F_{\mu \nu} F^{\mu \nu}$.

Here $F_{\mu \nu}$ is the Maxwell tensor. The scalar function $f(\phi)$ in (4) governs the non-trivial coupling between the supported scalar configurations and the electromagnetic field of the charged spacetime. This coupling function is characterized by the universal functional behavior $[9,10]$

$f(\phi)=1+\alpha \phi^{2}$

in the physical regime of linearized supported scalar fields. Here the dimensionless physical parameter $\alpha$ determines the strength of the non-minimal coupling between the scalar fields and the electromagnetic field of the charged spacetime. We shall henceforth assume $\alpha>0$.

Varying the action (4) with respect to the scalar field, one obtains the linearized scalar equation $[9,10]$

$\nabla^{\nu} \nabla_{\nu} \phi=\frac{1}{4} f_{, \phi} \mathcal{I}$.

It proves useful to use the mathematical decomposition [19]

$\phi(r, \theta, \phi)=\sum_{l m} R_{l m}(r) Y_{l m}(\theta) e^{i m \phi}$

for the supported field configurations, in terms of which one obtains from Eqs. (1), (2), and (7) the radial differential equation

$\frac{1}{r^{2}} \frac{d}{d r}\left[r^{2} h(r) \frac{d R}{d r}\right]-\left[\frac{l(l+1)}{r^{2}}-\frac{\alpha Q^{2}}{r^{4}}\right] R=0$.

The differential equation (9) determines the radial functional behavior of the supported non-minimally coupled scalar field configurations in the curved spacetime (1) of the supporting Reissner-Nordström black hole.

Below we shall use analytical techniques in order to solve the radial differential equation (9) for the spatially regular bound-state scalar configurations in the supporting charged black-hole spacetime (1). In particular, following $[9,10]$ we shall consider supported scalar eigenfunctions which are regular on the outer black-hole horizon and vanish asymptotically. These physically motivated boundary conditions are described by the simple functional relations $[9,10]$

$\phi\left(r=r_{+}\right)<\infty$ 
and

$\phi(r \rightarrow \infty) \rightarrow 0$.

The radial differential equation (9), supplemented by the boundary conditions (10) and (11), single out an infinitely large discrete resonant spectrum $\{\alpha(l, Q / M ; n)\}_{n=1}^{n=\infty}$ for the dimensionless coupling parameter $\alpha$ which characterizes the composed Reissner-Nordström-black-hole-nonminimallycoupled-massless-scalar-field configurations.

As we shall explicitly show below, for near-extremal (highly charged, $Q \simeq M$ ) central supporting ReissnerNordström black holes in the dimensionless regime $\left(r_{+}-\right.$ $\left.r_{-}\right) / r_{+} \ll 1$, the characteristic resonance spectrum $\{\alpha(l$, $Q / M ; n)\}$ that characterizes the critical existence-line of the composed black-hole-scalar-field system can be determined analytically [20].

\section{The resonance equation of the black-hole-scalar-field critical existence-line}

In the present section we shall use analytical techniques in order to study the radial differential equation (9) which, together with the boundary conditions (10) and (11), determines the characteristic radial eigenfunctions of the nonminimally coupled scalar field in the charged ReissnerNordström black-hole spacetime (1). In particular, we shall derive a resonance equation [see Eq. (37) below] for the dimensionless coupling parameter $\alpha$ which characterizes the critical existence-line of the non-minimally coupled Einstein-Maxwell-scalar theory (4) in the regime 1 $Q / M \ll 1$ of near-extremal central supporting black holes.

It is convenient to express the physical quantities that characterize the composed Reissner-Nordström-black-holenonminimally-coupled-massless-scalar-field system in terms of the dimensionless variables [21-23]

$x \equiv \frac{r-r_{+}}{r_{+}} ; \quad \tau \equiv \frac{r_{+}-r_{-}}{r_{+}}$.

Substituting the dimensionless variables $\{x, \tau\}$ from Eq. (12) into Eq. (9), one obtains the radial differential equation

$x(x+\tau) \frac{d^{2} R}{d x^{2}}+(2 x+\tau) \frac{d R}{d x}+U R=0$,

which determines the spatial behavior of the non-minimally coupled scalar eigenfunctions in the Reissner-Nordström black-hole spacetime, where

$U=U(x ; l, M, Q)=-l(l+1)+\frac{\alpha Q^{2}}{\left[r_{+}(x+1)\right]^{2}}$.
As we shall now prove explicitly, the ordinary differential equation (13) for the non-minimally coupled scalar field configurations can be solved analytically in the two asymptotic regions $x \ll 1$ and $x \gg \tau$ [24]. Interestingly, and most importantly for our analysis, in the regime

$\tau \ll 1$

of near-extremal central supporting black holes, one can use a standard matching procedure in the overlapping radial region $\tau \ll x \ll 1$ in order to determine the resonant spectrum $\{\alpha(l, Q, M ; n)\}_{n=1}^{n=\infty}$ of the dimensionless coupling parameter $\alpha$ which characterizes the composed ReissnerNordström-black-hole-nonminimally-coupled-masslessscalar-field configurations.

We shall first analyze the behavior of the radial scalar eigenfunctions in the region

$x \ll 1$.

In this near-horizon region, one can use in Eq. (14) the approximation $U \rightarrow U_{\text {near }} \equiv-l(l+1)+\alpha Q^{2} / r_{+}^{2}$ for the effective radial potential of the composed black-hole-scalarfield system. Defining

$z \equiv-\frac{x}{\tau}$

one obtains from (13) the near-horizon radial equation

$z(1-z) \frac{d^{2} R}{d z^{2}}+(1-2 z) \frac{d R}{d z}-U_{\text {near }} R=0$.

This is the familiar hypergeometric differential equation (see Eq. 15.5.1 of [25]). The near-horizon radial solution of (18) that respects the boundary condition (10) at the outer horizon of the central supporting Reissner-Nordström black hole is given by [24-29]

$R(x)={ }_{2} F_{1}\left(\frac{1}{2}+i \delta, \frac{1}{2}-i \delta ; 1 ;-\frac{x}{\tau}\right)$,

where ${ }_{2} F_{1}(a, b ; c ; z)$ is the familiar hypergeometric function and [30]

$\delta^{2} \equiv-\left(l+\frac{1}{2}\right)^{2}+\frac{\alpha Q^{2}}{r_{+}^{2}}$.

It proves useful to write the near-horizon eigenfunction (19) of the non-minimally coupled scalar field in the form (see Eq. 15.3.7 of [25]) 


$$
\begin{aligned}
R(x)= & \frac{\Gamma(2 i \delta)}{[\Gamma(1 / 2+i \delta)]^{2}}\left(\frac{x}{\tau}\right)^{-\frac{1}{2}+i \delta} \\
& \times{ }_{2} F_{1}\left(\frac{1}{2}-i \delta, \frac{1}{2}-i \delta ; 1-2 i \delta ;-\frac{\tau}{x}\right) \\
& +(\delta \rightarrow-\delta),
\end{aligned}
$$

where the notation $(\delta \rightarrow-\delta)$ means "replace $\delta$ by $-\delta$ in the preceding term". Using the characteristic asymptotic behavior (see Eq. 15.1.1 of [25])

${ }_{2} F_{1}(a, b ; c ; z) \rightarrow 1$ for $\frac{a b}{c} \cdot z \rightarrow 0$

of the hypergeometric function, one finds from (21) the simple functional behavior

$R(x)=\frac{\Gamma(2 i \delta) \tau^{1 / 2-i \delta}}{[\Gamma(1 / 2+i \delta)]^{2}} x^{-\frac{1}{2}+i \delta}+(\delta \rightarrow-\delta)$

for the radial eigenfunction of the non-minimally coupled scalar field in the intermediate radial region

$\tau \ll x \ll 1$.

We shall next analyze the behavior of the radial scalar eigenfunction in the region

$$
x \gg \tau \text {. }
$$

In this radial region one can replace Eq. (13) by

$x^{2} \frac{d^{2} R}{d x^{2}}+2 x \frac{d R}{d x}+U R=0$.

It proves useful to define the radial scalar eigenfunction

$S(x) \equiv x R(x)$,

in terms of which the radial equation (26) for the nearextremal-Reissner-Nordström-black-hole-nonminimallycoupled-massless-scalar-field system can be written as the Schrödinger-like ordinary differential equation [31]

$\frac{d^{2} S}{d x^{2}}-\left[\frac{l(l+1)}{x^{2}}-\frac{\alpha}{x^{2}(x+1)^{2}}\right] S(x)=0$.

Interestingly, and most importantly for our analysis, the differential equation (28) can be solved analytically. In particular, the solution of the Schrödinger-like differential equation (28) that respects the asymptotic boundary condition (11) is given by $[25,26,32,33]$

$$
\begin{aligned}
R(x)= & N_{1} \times x^{-\frac{3}{2}-l-i \sqrt{\alpha-1 / 4}}(x+1)^{\frac{1}{2}+i \sqrt{\alpha-1 / 4}}{ }_{2} F_{1} \\
& \times\left(i \sqrt{\alpha-1 / 4}+i \delta+l+1, i \sqrt{\alpha-1 / 4}-i \delta+l+1 ; 2 l+2 ;-\frac{1}{x}\right) .
\end{aligned}
$$

Using Eq. 15.3.7 of [25] and the simple asymptotic behavior (22) of the hypergeometric function, one finds the asymptotic functional behavior

$$
\begin{aligned}
R(x)= & N_{1} \times \frac{\Gamma(2 l+2) \Gamma(-2 i \delta)}{\Gamma(i \sqrt{\alpha-1 / 4}-i \delta+l+1) \Gamma(-i \sqrt{\alpha-1 / 4}-i \delta+l+1)} \\
& x^{-\frac{1}{2}+i \delta}+(\delta \rightarrow-\delta)
\end{aligned}
$$

of the scalar eigenfunction (29) in the intermediate radial region

$\tau \ll x \ll 1$

Interestingly, for near-extremal Reissner-Nordström black holes in the dimensionless physical regime $\tau \ll 1$, there is an overlap radial region,

$\tau \ll x_{o} \ll 1$,

in which the functional expressions (23) and (30) for the spatial behavior of the non-minimally coupled scalar field are both valid. Note, in particular, that in terms of the dimensionless coordinate $x$, the analytically derived radial expressions (23) and (30) for the scalar eigenfunction in the overlap region (32) are characterized by the same functional behavior. Matching the radial expressions (23) and (30) in their overlap radial region (32), one finds the relations

$$
\begin{aligned}
N_{1} & \times \frac{\Gamma(2 l+2) \Gamma(-2 i \delta)}{\Gamma(i \sqrt{\alpha-1 / 4}-i \delta+l+1) \Gamma(-i \sqrt{\alpha-1 / 4}-i \delta+l+1)} \\
& =\frac{\Gamma(2 i \delta) \tau^{1 / 2-i \delta}}{[\Gamma(1 / 2+i \delta)]^{2}}
\end{aligned}
$$

and

$$
\begin{aligned}
N_{1} & \times \frac{\Gamma(2 l+2) \Gamma(2 i \delta)}{\Gamma(i \sqrt{\alpha-1 / 4}+i \delta+l+1) \Gamma(-i \sqrt{\alpha-1 / 4}+i \delta+l+1)} \\
& =\frac{\Gamma(-2 i \delta) \tau^{1 / 2+i \delta}}{[\Gamma(1 / 2-i \delta)]^{2}} .
\end{aligned}
$$

From Eqs. (33) and (34) one obtains the resonance equation

$$
\begin{aligned}
{\left[\frac{\Gamma(-2 i \delta)}{\Gamma(2 i \delta)}\right]^{2}\left[\frac{\Gamma(1 / 2+i \delta)}{\Gamma(1 / 2-i \delta)}\right]^{2} } & \\
& \frac{\Gamma(i \sqrt{\alpha-1 / 4}+i \delta+l+1) \Gamma(-i \sqrt{\alpha-1 / 4}+i \delta+l+1)}{\Gamma(i \sqrt{\alpha-1 / 4}-i \delta+l+1) \Gamma(-i \sqrt{\alpha-1 / 4}-i \delta+l+1)} \tau^{2 i \delta}=1
\end{aligned}
$$

for the composed black-hole-field system. Using the characteristic relation (see Eq. 6.1.18 of [25])

$$
\Gamma(2 z)=(2 \pi)^{-\frac{1}{2}} 2^{2 z-\frac{1}{2}} \Gamma(z) \Gamma\left(z+\frac{1}{2}\right)
$$


for the Gamma function, one can express (35) in the form $[34,35]$

$\left[\frac{\Gamma(-i \delta)}{\Gamma(i \delta)}\right]^{2} \frac{\Gamma(i \sqrt{\alpha-1 / 4}+i \delta+l+1) \Gamma(-i \sqrt{\alpha-1 / 4}+i \delta+l+1)}{\Gamma(i \sqrt{\alpha-1 / 4}-i \delta+l+1) \Gamma(-i \sqrt{\alpha-1 / 4}-i \delta+l+1)}\left(\frac{\tau}{16}\right)^{2 i \delta}$ $=1$.

The analytically derived resonance equation (37) determines the characteristic resonance spectrum $\{\alpha(l, Q$, $M ; n)\}_{n=1}^{n=\infty}$ of the composed near-extremal-Reissner-Nordström-black-hole-nonminimally-coupled-massless-scalarfield configurations. In the next section we shall explicitly prove that the (rather cumbersome) resonance equation (37) can be solved analytically in the dimensionless regime $\delta \ll 1$ which characterizes the near-extremal $(\tau \ll 1)$ central supporting Reissner-Nordström black holes.

\section{The resonance spectrum of the composed Reissner-Nordström-black-hole-nonminimally- coupled-massless-scalar-field configurations}

Interestingly, in the present section we shall explicitly show that the resonance equation (37) for the dimensionless physical parameter $\alpha$ can be solved analytically in the regime

$\delta \ll 1$.

As we shall show below, the regime (38) corresponds to the case of near-extremal $(\tau \ll 1)$ central supporting black holes [see Eq. (42) below].

In the $\delta \ll 1$ regime, one can expand the left-hand side of (37) in the form

$\left[1+2 i c_{l} \cdot \delta+O\left(\delta^{2}\right)\right]\left(\frac{\tau}{16}\right)^{2 i \delta}=1$

where

$c_{l}=2 \gamma+\psi[l+1+i \sqrt{l(l+1)}]+\psi[l+1-i \sqrt{l(l+1)}]$.

Here $\gamma$ is the Euler constant and $\psi(z)$ is the Psi (Digamma) function $[25,36]$. Taking the logarithm of both sides of (39), one obtains the equation [37]

$2 i c_{l} \delta+2 i \delta \cdot(\ln \tau-4 \ln 2)=-2 i \pi n \quad ; \quad n=1,2,3, \ldots$

which yields the simple small- $\delta(\tau \ll 1)$ relation

$$
\delta(\tau, l ; n)=\frac{\pi n}{|\ln \tau|+4 \ln 2-c_{l}} .
$$

Note that one finds, in accord with the assumed strong inequality (38), the relation $\delta \ll 1$ in the regime $\tau \ll 1$ $(|\ln \tau| \gg 1)$ of near-extremal black holes [38].

Taking cognizance of Eqs. (20) and (42), one finds the remarkably compact resonance formula [31]

$$
\begin{aligned}
\alpha(l, \tau ; n) & =\left(l+\frac{1}{2}\right)^{2}+\left(\frac{\pi}{|\ln \tau|+4 \ln 2-c_{l}}\right)^{2} \cdot n^{2} \\
n & =1,2,3, \ldots
\end{aligned}
$$

for the dimensionless coupling parameter $\alpha$ which characterizes the critical existence-line of the composed nearextremal-Reissner-Nordström-black-hole-nonminimallycoupled-massless-scalar-field configurations [39].

\section{Spherically symmetric non-minimally coupled scalar clouds}

Intriguingly, it has been proved in the physically important works $[9,10]$ that, for spherically symmetric composed black-hole-scalar-field configurations, the critical existenceline of the system (which corresponds to supported linearized scalar clouds with $l=0$ ) is determined by the mathematically compact resonance equation

${ }_{2} F_{1}\left[1 / 2-i \sqrt{\alpha-1 / 4}, 1 / 2+i \sqrt{\alpha-1 / 4} ; 1 ; x^{2} /\left(x^{2}-1\right)\right]=0$,

where $x \equiv Q / r_{+}$. This resonance equation for the spherically symmetric Reissner-Nordström-black-hole-nonminimally-coupled-linearized-massless-scalar-field configurations has been solved numerically in $[9,10]$. Interestingly, it has been proved in [17] that, for near-extremal (highly charged) central supporting black holes, the $l=0$ resonance equation (44) can be solved analytically. In particular, one finds the compact resonance formula [17]

$\alpha_{n}=\frac{1}{4}+\left[\frac{2 \pi n}{\ln \left(\frac{1-\bar{Q}}{32}\right)}\right]^{2} ; \quad n=1,2,3, .$.

for the composed near-extremal-black-hole-nonminimallycoupled-spherically-symmetric-massless-scalar-field cloudy configurations in the dimensionless physical regime

$1-\bar{Q} \ll 1$

where $\bar{Q} \equiv Q / M$.

It is of physical interest to test the accuracy of our analytical matching procedure using the accurate resonance formula $(45)$ of $[9,10,17]$ for the spherically symmetric $(l=0)$ [40] supported scalar clouds. In the present section we shall 
explicitly prove that the resonance spectra (43) and (45) agree in the $l=0$ case.

To this end, we first note that $\psi(1)=-\gamma$ (see Eq. 6.3.2 of [25]), which yields the simple relation [see Eq. (40)]

$c_{0}=0$.

Substituting (47) into the analytically derived resonance formula (43) and using the relation [41]

$\tau=2 \sqrt{2} \sqrt{1-\bar{Q}} \cdot[1+O(\sqrt{1-\bar{Q}})]$

for near-extremal Reissner-Nordström black holes in the regime (15), one finds

$$
\begin{gathered}
\alpha(l=0, \bar{Q} \rightarrow 1 ; n)=\frac{1}{4}+\left[\frac{\pi}{\ln (2 \sqrt{2} \sqrt{1-\bar{Q}})-4 \ln 2}\right]^{2} \\
\cdot n^{2} ; \quad n=1,2,3, \ldots
\end{gathered}
$$

for the spherically symmetric scalar clouds. The expression (49) can also be written in the form

$\alpha(l=0, \bar{Q} \rightarrow 1 ; n)=\frac{1}{4}+\left[\frac{\pi n}{\ln \left(\sqrt{\frac{1-\bar{Q}}{32}}\right)}\right]^{2} ; n=1,2,3, \ldots$

which coincides with the resonance spectrum (45) of the spherically symmetric $(l=0)$ scalar clouds [40] that has been derived in [17] from the exact resonance equation (44) of $[9,10]$. This agreement provides compelling evidence for the validity of our analytically derived resonance spectrum (43).

\section{Summary}

Recent studies of the coupled Einstein-Maxwell-scalar equations $[9,10]$ have revealed the physically important fact that asymptotically flat charged black-hole spacetimes can support scalar field configurations which are non-minimally coupled to the electromagnetic Maxwell tensor. As explicitly shown in $[9,10]$, the composed black-hole-scalar-field hairy configurations exist in the non-trivially coupled EinsteinMaxwell-scalar theory (4) provided the dimensionless coupling parameter $\alpha$ of the theory is larger than a critical value $\alpha_{\min }(Q / M, l)$ that depends on the dimensionless charge-tomass ratio $Q / M$ of the central supporting black hole and on the angular harmonic parameter $l$ of the supported nonminimally coupled scalar fields.

In particular, it has been revealed in $[9,10]$ that the critical boundary between bald (scalarless) charged black-hole solutions of the field equations and hairy charged-black-holenonminimally-coupled-massless-scalar-field configurations is marked by the presence of a critical existence-line. This critical boundary corresponds to spatially regular linearized non-minimally coupled scalar field configurations (scalar 'clouds') that are supported by central Reissner-Nordström black holes.

In the present paper we have explicitly proved that, for near-extremal central supporting black holes, the physical and mathematical properties of the composed charged-blackhole-nonminimally-coupled-linearized-massless-scalar-field cloudy configurations can be studied analytically for generic values of the angular harmonic parameter $l$ [42]. In particular, using a matching procedure in the regime $\tau \ll 1$ of near-extremal black holes, we have derived the remarkably compact $l$-dependent analytical formula [see Eqs. (40) and $(43)][43,44]$

$\alpha_{\min }(l, \tau)=\left(l+\frac{1}{2}\right)^{2}+\left(\frac{\pi}{|\ln \tau|+4 \ln 2-c_{l}}\right)^{2}$

for the dimensionless physical parameter $\alpha$ of the nonminimally coupled Einstein-Maxwell-scalar theory (4). The resonance formula (51) characterizes the critical existenceline of the composed black-hole-field system in the regime (15) of near-extremal central supporting black holes.

Finally, it is worth emphasizing again that the physical significance of the analytically derived resonance formula (51) stems from the fact that, for given values $\{l, \tau\}$ of the blackhole-field physical parameters, the composed charged-blackhole-nonminimally-coupled-massless-scalar-field hairy configurations are characterized by the critical relation $\alpha(l, \tau) \geq$ $\alpha_{\min }(l, \tau)$.

Acknowledgements This research is supported by the Carmel Science Foundation. I thank Yael Oren, Arbel M. Ongo, Ayelet B. Lata, and Alona B. Tea for stimulating discussions.

Data Availability Statement This manuscript has no associated data or the data will not be deposited. [Authors' comment: There are no external data associated with the manuscript.]

Open Access This article is licensed under a Creative Commons Attribution 4.0 International License, which permits use, sharing, adaptation, distribution and reproduction in any medium or format, as long as you give appropriate credit to the original author(s) and the source, provide a link to the Creative Commons licence, and indicate if changes were made. The images or other third party material in this article are included in the article's Creative Commons licence, unless indicated otherwise in a credit line to the material. If material is not included in the article's Creative Commons licence and your intended use is not permitted by statutory regulation or exceeds the permitted use, you will need to obtain permission directly from the copyright holder. To view a copy of this licence, visit http://creativecomm ons.org/licenses/by/4.0/.

Funded by SCOAP ${ }^{3}$. 


\section{References}

1. J.D. Bekenstein, Phys. Rev. D 5, 1239 (1972)

2. C.A.R. Herdeiro, E. Radu, Int. J. Mod. Phys. D 24, 1542014 (2015)

3. S. Hod, Phys. Lett. B 713, 505 (2012)

4. S. Hod, Phys. Lett. B 718, 1489 (2013). arXiv:1304.6474

5. S. Hod, Phys. Rev. D 91, 044047 (2015). arXiv:1504.00009

6. A.E. Mayo, J.D. Bekenstein, Phys. Rev. D 54, 5059 (1996)

7. S. Hod, Phys. Lett. B 771, 521 (2017)

8. S. Hod, Phys. Rev. D 96, 124037 (2017)

9. C.A.R. Herdeiro, E. Radu, N. Sanchis-Gual, J.A. Font, Phys. Rev. Lett. 121, 101102 (2018)

10. P.G.S. Fernandes, C.A.R. Herdeiro, A.M. Pombo, E. Radu, N. Sanchis-Gual, Class. Quantum Gravity 36, 134002 (2019). arXiv: 1902.05079

11. Here $Q / M$ is the dimensionless charge-to-mass ratio of the central supporting black hole and the integer $l \geq 0$ is the angular harmonic index of the supported scalar mode [see Eq. (8) below]

12. We shall henceforth assume $Q>0$ without loss of generality

13. S. Hod, Phys. Rev. D 86, 104026 (2012). arXiv: 1211.3202

14. S. Hod, Eur. Phys. JouReissner-Nordströmal C 73, 2378 (2013). arXiv: 1311.5298

15. S. Hod, Phys. Rev. D 90, 024051 (2014). arXiv:1406.1179

16. C.A.R. Herdeiro, E. Radu, Phys. Rev. Lett. 112, 221101 (2014)

17. S. Hod, Phys. Lett. B 798, 135025 (2019). arXiv:2002.01948

18. We shall use natural units in which $G=c=1$

19. The dimensionless integer parameters $\{l, m\}$ of the scalar field modes in (8) are the angular harmonic indices. In particular, the characteristic angular eigenvalue of the angular scalar function $Y_{l m}(\theta)$ is given by the simple expression $l(l+1)$. For brevity, we shall henceforth omit the angular parameters $\{l, m\}$ of the scalar field modes

20. It is worth stressing again that the physical significance of the critical existence-line $\alpha=\alpha(Q / M ; l)$ stems from the fact that, for a given value $l$ of the angular harmonic index of the supported nonminimally coupled scalar field, this existence-line marks the boundary between bald (scalarless) Reissner-Nordström black holes and hairy charged-black-hole-nonminimally-coupled-massless-scalarfield configurations of the Einstein-Maxwell-scalar theory (4)

21. S.A. Teukolsky, Phys. Rev. Lett. 29, 1114 (1972)

22. S.A. Teukolsky, Astrophys. J. 185, 635 (1973)

23. T. Hartman, W. Song, A. Strominger, JHEP 1003, 118 (2010)

24. S. Hod, O. Hod, Phys. Rev. D 81, Rapid communication 061502 (2010) arXiv:0910.0734

25. M. Abramowitz, I.A. Stegun, Handbook of Mathematical Functions (Dover Publications, New York, 1970)

26. P.M. Morse, H. Feshbach, Methods of Theoretical Physics (McGraw-Hill, New York, 1953)

27. See, in particular, Eqs. 15.5.16 and 15.5.17 of [25]

28. Without loss of generality, we have chosen the normalization constant of the linear eigenfunction (19) to be 1

29. Using the characteristic asymptotic behavior ${ }_{2} F_{1}(a, b ; c ; z) \rightarrow 1$ for $(a b / c) \cdot z \rightarrow 0$ of the hypergeometric function (see Eq. 15.1.1 of [25]), one finds that the radial scalar eigenfunction (19) is well behaved at the outer black-hole horizon. In particular, $R(x \rightarrow$ 0) $\rightarrow 1$

30. We shall henceforth assume that $\delta \neq 0$

31. Here we have used the relation $Q / r_{+} \rightarrow 1$ for the near-extremal central supporting Reissner-Nordström black hole

32. Here we have used Eq. 15.5.8 of [25]

33. Using the characteristic asymptotic behavior (22) of the hypergeometric function, one finds that the scalar eigenfunction (29) decays asymptotically as $R(x \rightarrow \infty) \sim x^{-l-1}$

34. As a consistency check, we note that one finds from equation 6.1 .23 of [25] that $\Gamma(-i \delta) / \Gamma(i \delta)=e^{i \phi_{1}}, \Gamma(i \sqrt{\alpha-1 / 4}+i \delta+l+$
1) $/ \Gamma(-i \sqrt{\alpha-1 / 4}-i \delta+l+1)=e^{i \phi_{2}}$, and $\Gamma(-i \sqrt{\alpha-1 / 4}+i \delta+$ $l+1) / \Gamma(i \sqrt{\alpha-1 / 4}-i \delta+l+1)=e^{i \phi_{3}}$, where $\left\{\phi_{1}, \phi_{2}, \phi_{3}\right\} \in \mathbb{R}$ for $\delta \in \mathbb{R}$. These relations imply that the absolute value of the left-hand-side of (37) equals 1 , as should be the case

35. The authors of the physically important work [9] have reported the values $\alpha(l=1)=2.784$ and $\alpha(l=2)=7.087$ for the dimensionless coupling parameter of the composed Einstein-Maxwell-scalar theory (4) in the regime (15) of near-extremal central supporting Reissner-Nordström black holes. We have substituted these numerically computed values into the analytically derived resonance formula (37) and found that both these values correspond to highlycharged black holes with $1-Q / M \simeq 5 \times 10^{-5} \ll 1$

36. It is interesting to note that the expansion coefficient $c_{l}$ is a monotonic increasing function of the angular harmonic index $l$ of the supported non-minimally coupled scalar clouds. In particular, from Eq. (40) one finds $c_{0}=0, c_{1}=2.603, c_{2}=3.660, c_{3}=4.343$ for the first few values of the dimensionless coefficient $c_{l}$. In the $l \rightarrow \infty$ limit one finds $c_{l}=2 \ln (l)+\ln 2+2 \gamma+O\left(l^{-1}\right)$

37. Here we have used the relation $1=e^{-2 i \pi n}$, where the resonance parameter $n$ is an integer

38. Note that $c_{l}=2 \ln (l)+O(1)$ in the large- $l$ regime [36], which implies that our analytical treatment for the $\delta \ll 1$ case is valid for near-extremal black holes in the dimensionless regime $\tau \ll$ $(l+1)^{-2} e^{-n \pi}$

39. It is interesting to note that the resonance formula (43) reveals the fact that the larger is the value of the angular harmonic index $l$, the slower is the approach of the dimensionless coupling parameter of the theory to its extremal limit $\alpha(\tau \rightarrow 0 ; l) \rightarrow l(l+1)$ (this observation stems from the fact that the coefficient $c_{l}$ is a monotonically increasing function of $l[36]$ )

40. It is worth emphasizing again that the validity of the resonance formula (45) is restricted to the case of spherically symmetric $l=0$ scalar clouds, whereas the analytically derived resonance formula (43) of the present paper is valid for generic values of the angular harmonic index $l$ of the supported scalar clouds

41. Here we have used the relations $\tau \equiv\left(r_{+}-r_{-}\right) / r_{+}=$ $2 \sqrt{M^{2}-Q^{2}} /\left(M+\sqrt{M^{2}-Q^{2}}\right)=2 \sqrt{1-\bar{Q}^{2}} /\left(1+\sqrt{1-\bar{Q}^{2}}\right)=$ $2 \sqrt{(1-\bar{Q})(1+\bar{Q})} /\left(1+\sqrt{1-\bar{Q}^{2}}\right)=2 \sqrt{2} \sqrt{1-\bar{Q}} \cdot[1+$ $O(\sqrt{1-\bar{Q}})]$ for charged Reissner-Nordström black holes in the near-extremal $1-\bar{Q} \ll 1$ regime

42. It is worth noting again that the physical and mathematical properties of spherically-symmetric $(l=0)$ Reissner-Nordström-blackhole-nonminimally-coupled-massless-scalar-field cloudy configurations have been studied analytically in $[9,10,17]$. In the present paper we have explicitly proved that, in the regime (15) of nearextremal central supporting black holes, the physical and mathematical properties of these intriguing black-hole-field configurations can be studied analytically for generic values of the angular harmonic index $l$ which characterizes the cloudy field configurations

43. Note that the critical existence-line, which separates bald ReissnerNordström black-hole spacetimes from hairy charged-black-holenonminimally-coupled-massless-scalar-field configurations, corresponds to the fundamental $(n=1)$ resonant mode of the analytically derived discrete resonance spectrum (43)

44. It is worth emphasizing again that, for a given value of the dimensionless angular harmonic index $l$, the composed black-holelinearized-field configurations are characterized by an infinitely large discrete resonance spectrum $\{\alpha(Q / M ; l)\}_{n=1}^{n=\infty}$ [see Eq. (43)] of the physical coupling parameter $\alpha$ of the theory $[9,10]$ 